\title{
Ecriture de soi et lecture religieuse chez les protestants du monde germanophone en perspective confessionnelle
}

\section{Kaspar Von Greyerz}

Traducteur : Danièle Tosato-Rigo

\section{(2) OpenEdition}

\section{Journals}

Édition électronique

URL : http://journals.openedition.org/edl/863

DOI : $10.4000 /$ edl. 863

ISSN : 2296-5084

\section{Éditeur}

Université de Lausanne

\section{Édition imprimée}

Date de publication : 15 mai 2016

ISBN : 978-2-940331-48-2

ISSN : 0014-2026

\section{Référence électronique}

Kaspar Von Greyerz, «Ecriture de soi et lecture religieuse chez les protestants du monde

germanophone en perspective confessionnelle », Etudes de lettres [En ligne], 1-2 | 2016, mis en ligne le 01 mai 2019, consulté le 15 décembre 2020. URL : http://journals.openedition.org/edl/863 ; DOI : https://doi.org/10.4000/edl.863 


\section{ECRITURE DE SOI ET LECTURE RELIGIEUSE CHEZ LES PROTESTANTS DU MONDE GERMANOPHONE EN PERSPECTIVE CONFESSIONNELLE}

Partant de l'expérience d'un projet de recherche ("Les écrits personnels, sources d'histoire des mentalités») et d'une base de données répertoriant des écrits personnels de l'époque moderne, l'auteur puise dans ce corpus pour proposer une lecture renouvelée des liens très discutés entre protestantisme et écriture personnelle. Il analyse quelques aspects importants des textes protestants - histoire du salut, martyre, providentialisme - avant de s'arrêter sur la question de la lecture religieuse, et de sa présence inégale chez des auteur-e-s catholiques et protestant-e-s. Ce faisant, il met en avant les difficultés d'interprétation que posent les egodocuments, soulignant le côté réducteur de tout fonctionnalisme face à un protestantisme pluriel et à la diversité des langages autobiographiques.

Les recherches concernant l'écriture de soi dans le monde germanophone ont été jusqu'à la fin des années 1980 et à quelques exceptions près l'affaire des germanistes et, plus rarement, des latinistes. Elles s'appuyaient largement sur un corpus bien défini de textes édités, répondant aux exigences méthodologiques - surtout de type esthétique - imparties à ces textes par les chercheurs en question.

C'est dans ce contexte qu'un groupe de trois doctorants (Gudrun Piller, Sebastian Leutert, Lorenz Heiligensetzer) et moi-même avons entrepris dans les années 1990 de nous lancer dans le dépouillement archivistique et l'étude de l'écriture de soi dans la Suisse de l'époque moderne, avec un intérêt particulier pour la question de sa répartition confessionnelle. S'y ajoutait le vœu d'augmenter le nombre de textes connus jusqu'ici rédigés par des femmes. Si ce dernier souhait n'a malheureusement pas pu être exaucé - les écrits féminins, principalement 
du XVIII e et début du XIXe siècle, ne représentant que $10 \%$ des textes répertoriés -, les liens entre protestantisme et écriture personnelle ont, quant à eux, pu être étudiés sous divers angles. Dans les lignes qui suivent, je tenterai, après un préambule de nature documentaire, d'esquisser quelques aspects des textes protestants que j'estime être importants, en marrêtant un peu plus longuement sur la question de la lecture religieuse, avant d'élargir la perspective au-delà du monde germanique en guise de conclusion.

\section{Une pesée archivistique}

Sur un plan général, la campagne de repérage d'écrits personnels entreprise dans le cadre du projet FNS ${ }^{1}$ "Selbstzeugnisse als Quellen der Mentalitätsgeschichte" nous a appris que les fonds d'archives et de bibliothèques ne nous informent pas sur la répartition réelle de l'écriture de soi entre hommes et femmes ou entre groupes sociaux ou confessionnels. Ces fonds ne nous fournissent en fin de compte que des renseignements sur la tradition locale ou régionale de la collecte de sources, comme sur le soin apporté - ou non - au patrimoine immatériel en certains endroits, tant au niveau local que régional.

Ainsi, lorsque sur près de 860 documents dépouillés dans les principales institutions de conservation publiques de Suisse alémanique par l'équipe bâloise, près du quart des scripteurs s'avèrent appartenir au clergé, et, à quelques exceptions près, au clergé réformé, qu'en déduire? Que l'écriture de soi de clercs catholiques était rare, ou bien qu'elle ne nous est pas parvenue. Ou encore, que ce genre de textes n'a jusqu'ici pas été dépouillé. A ce propos, Louis Châtellier, éminent connaisseur de l'histoire du catholicisme moderne, m'avait suggéré il y a quelques années de rechercher systématiquement de tels documents sur le plan communal, les prêtres catholiques ayant peut-être inséré leurs notes autobiographiques dans les registres de paroisse. Il s'agissait d'une hypothèse, et l'on ne peut guère en dire plus aujourd'hui puisqu'à ma connaissance personne ne s'est encore aventuré dans une recherche sur le plan communal.

I. Fonds national suisse de la recherche scientifique. 
Quoi qu'il en soit, la validité du constat relatif aux écrits personnels de prêtres suisses alémaniques à l'époque moderne peut être étendue plus généralement au monde germanique. Mais là encore, avant d'accepter les choses telles qu'elles se présentent à nous, il faudrait se demander dans quelle mesure il y eut une politique plus systématique de conservation et de dépouillement du côté protestant que du côté catholique, au $\mathrm{XIX}^{\mathrm{e}}$ siècle en particulier, lorsqu'on commença à s'intéresser à ce genre de textes à titre de sources historiques. De même qu'il importe de relever, dans le cas de la Suisse alémanique, que l'essentiel de l'écriture de soi émane d'un contexte urbain: or, la plupart des grandes villes - sauf Fribourg, Soleure et Lucerne - sont réformées. Cela montre que nous sommes confrontés, d'une part, à la problématique de l'alphabétisation des régions rurales et catholiques et, d'autre part, et c'est plus important encore, à celle de la conservation de l'héritage historique que constituent de tels textes dans ces régions. Quant à ce qui pourrait apparaître comme spécifique de l'écriture protestante, comment l'évaluer? Les réflexions qui suivent tentent d'apporter des éléments de réponse - souvent probatoires - à une telle question.

\section{Histoire du salut, martyre et haine}

Dans une contribution à un volume collectif consacré à l'attitude des individus des mondes médiéval et moderne vis-à-vis du changement de siècle, l'historien de l'église catholique Andreas Holzem s'est demandé pourquoi la signification du passage d'un siècle à l'autre ne préoccupait guère les théologiens germanophones catholiques vers 1700, alors qu'un grand nombre de traités protestants de la même période s'efforçaient de rendre compte de l'importance de l'événement dans le cadre d'une histoire collective du salut ${ }^{2}$. Encore peu concernée par cette dernière, supposait Holzem, la théologie catholique germanophone du XVII siècle aurait préféré mettre l'accent sur la théologie pastorale et sur le passage individuel d'un triste ici-bas vers un glorieux au-delà. Une interprétation à laquelle fait écho l'analyse du journal personnel tenu pendant la guerre de Trente Ans par l'abbé bénédictin Georg Gaisser, de Christian Schulz,

2. A. Holzem, «Zeit-Zeitenwende-Endzeit?». 
qui évoque la "posture fondamentalement fataliste» du prélat face aux troubles de son temps ${ }^{3}$.

Dans quelle mesure une telle différence se reflète-t-elle dans des textes autobiographiques du XVII ${ }^{\mathrm{e}}$ siècle? A première vue, si l'on prend en considération des récits autobiographiques relatifs aux années 1630, sensibles au climat de la guerre de Trente Ans qui sévissait au sud-ouest du Saint-Empire, force est de constater qu'il y a une unanimité supraconfessionelle dans l'évocation de l'imminence de la fin du monde. Rien ne distingue à cet égard, par exemple, le texte de la main de Hans Heberle, cordonnier d'un village près d'Ulm, de celui de Klara Staiger, abbesse d'un couvent à proximité d'Eichstätt en Bavière ${ }^{4}$. A y regarder de plus près cependant, on peut relever une différence de taille dans leur évocation de l'entrée en guerre de Gustave Adolphe de Suède. Alors que les auteurs protestants mettent clairement en évidence le rôle de cet événement dans leur conception de l'histoire du salut, on cherche en vain des passages analogues dans des textes catholiques. Certes, on pourrait arguer que les auteurs protestants se sont simplement ralliés au point de vue exprimé par des douzaines (voire des centaines) de feuilles volantes qui célébraient alors l'événement. Mais là n'est pas l'essentiel. Ce qui importe davantage c'est qu'un grand nombre d'auteurs protestants semble avoir adopté cette perspective historique avec un arrière-fond millénariste.

Ainsi, pour se limiter à trois exemples, Hans Heberle attribue au roi de Suède le rôle de sauveur du protestantisme. Mais pour le scripteur le metteur en scène est à l'évidence Dieu qui "peut opérer des fins et des changements en tout " 5 . Le potier d'étain Augustin Güntzer adopte un point de vue similaire, en louant Dieu d'avoir préservé ses fidèles contre la fureur de l'Antéchrist ${ }^{6}$. Et en Suisse, c'est en ces termes que le paysan emmentalois Jodocus Jost commente l'arrivée des Suédois en Poméranie:

Cela s'est produit en automne, lorsqu'on ne savait encore rien à propos des Suédois. C’est alors que le Seigneur opposa une résistance à tous

3. C. Schulz, «Das Strafgericht Gottes oder menschliches Versagen?», p. 279.

4. K. Staiger, Klara Staigers Tagebuch.

5. G. Zillhardt, Der Dreissigjährige Krieg in zeitgenössischer Darstellung, p. 130.

6. A. Güntzer, Kleines Biechlin von meinem gantzen Leben, p. 229; traduction française: L'histoire de toute ma vie, p. 170. Voir également: D. Sieber, "Calvinistische Passionen, konfessionalisierte Körper ", p. 9-11. 
les papistes par l'intermédiaire des Suédois, par la mer, en 1630, aux environs de carnaval 7 .

Dans le même ordre d'idées, je ne connais aucun équivalent catholique comparable aux "éruptions" apocalyptiques que l'on rencontre dans la chronique d'Augsbourg de 1576 à 1607 de Georg Kölderer ${ }^{8}$ qui, à l'instar de celle de Jodocus Jost, n'est pas seulement une chronique dans le sens strict du terme, mais également une "écriture de soi».

Au même titre que l'histoire du salut, la souffrance exemplaire et le martyre pourraient être retenus, à titre d'hypothèse pour le moins, comme point saillant d'écrits personnels protestants.

J'en donnerai pour exemple le pasteur Johannes Erb (1635-1701). L'homme d'église se présente comme martyr dans le titre même de son autobiographie:

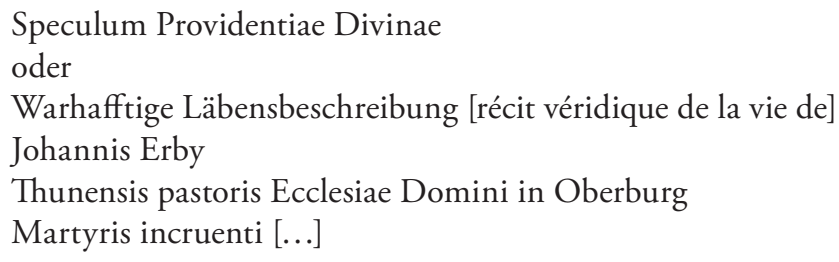

Né à Thoune en 1635, Erb a étudié à Berne et Heidelberg avant d'officier comme ministre de l'église réformée dans le canton de Berne - d'abord à Grindelwald, dans l'Oberland, puis à Oberburg, près de Berthoud - où il eut des démêlés non seulement avec ses ouailles, mais avec les autorités locales ${ }^{9}$. Il s'est fait un nom comme traducteur de traités d'édification parus en anglais et en néerlandais. Son autobiographie, inédite, revêt la forme d'un dialogue entre deux amis: Armgast (le "convive pauvre») et Baldreich ( bientôt riche»). On peut sans peine identifier Armgast comme l'alter ego de l'auteur, pauvre dans ce monde qui le traite d'intrus. Quant à l'expression de "Martyris incruenti", elle se réfère à un martyre sans effusion de sang.

7. D. Tosato-Rigo, La chronique de Jodocus Jost, p. 211 sq., traduit de l'allemand.

8. G. Kölderer, Beschreibung vnnd Kurtze Vertzaichnus Fürnemer Lob vnnd gedenckhwürdiger Historien.

9. Voir L. Heiligensetzer, Getreue Kirchendiener - gefährdete Pfarrherren, p. 218227 et "Swiss-German Self-Narratives", p. 68-73. 


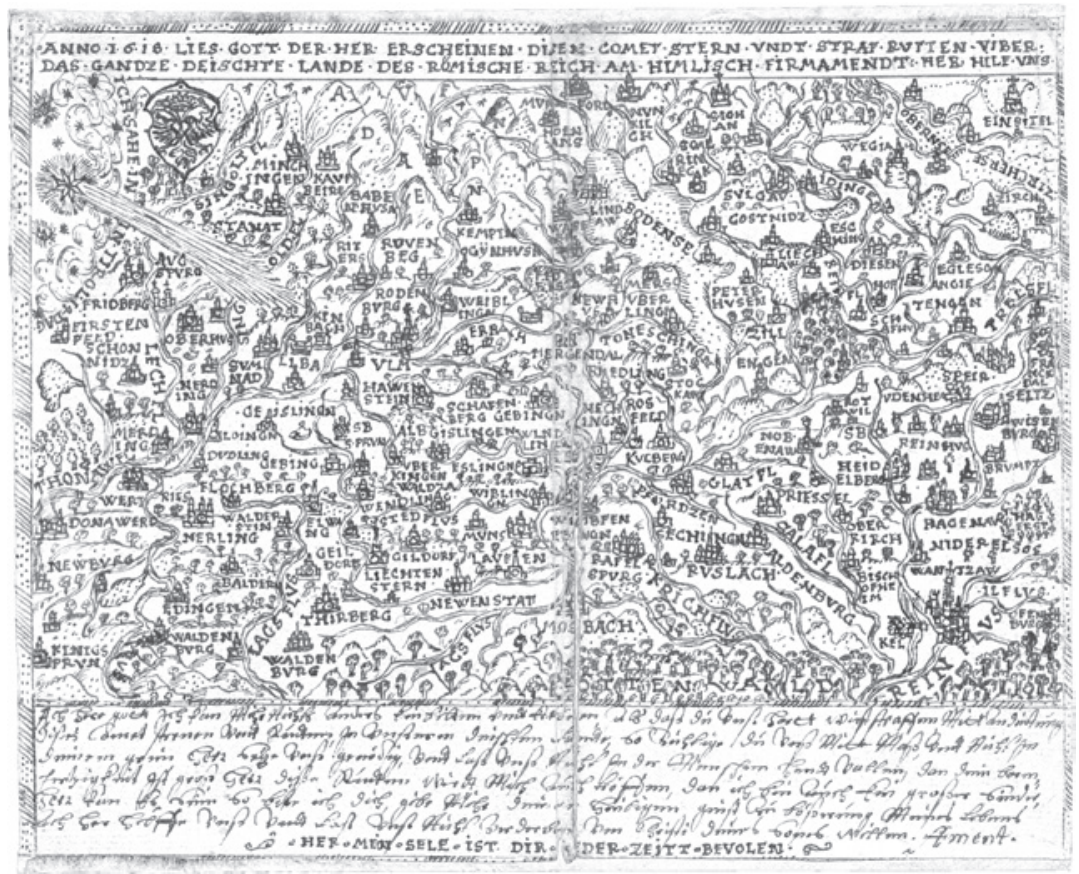

Fig. 1 - La comète de 1618 menaçant le sud de l'Allemagne (dessin d'Augustin Güntzer)

Une telle stylisation a ceci de particulier que pour Johannes Erb il était tout à fait possible de devenir un martyr parmi ses propres coreligionnaires, alors que d'autres textes du XVII ${ }^{e}$ siècle ne conçoivent de telles souffrances que dans le cadre de tensions entre membres de confessions différentes. C'est le cas de celui d'Augustin Güntzer, mentionné précédemment. L'auteur est marqué dès son enfance par l'expérience de la marginalité confessionnelle, en tant que calviniste dans un environnement d'abord majoritairement catholique, puis luthérien. Son autobiographie contient une profession de foi calviniste très détaillée. Et pourtant, sa curiosité incite le potier d'étain à visiter notamment la chapelle de Notre-Dame de Lorette et la Basilique Saint-Pierre de Rome. En portant sur lui un chapelet, il tente de se protéger des accusations confessionnelles. Mieux encore: à Sienne, comme cent cinquante autres croyants, il se fait laver les pieds par un évêque, et en 1621, au retour d'un deuxième voyage de compagnonnage, il se signe pour échapper à la 


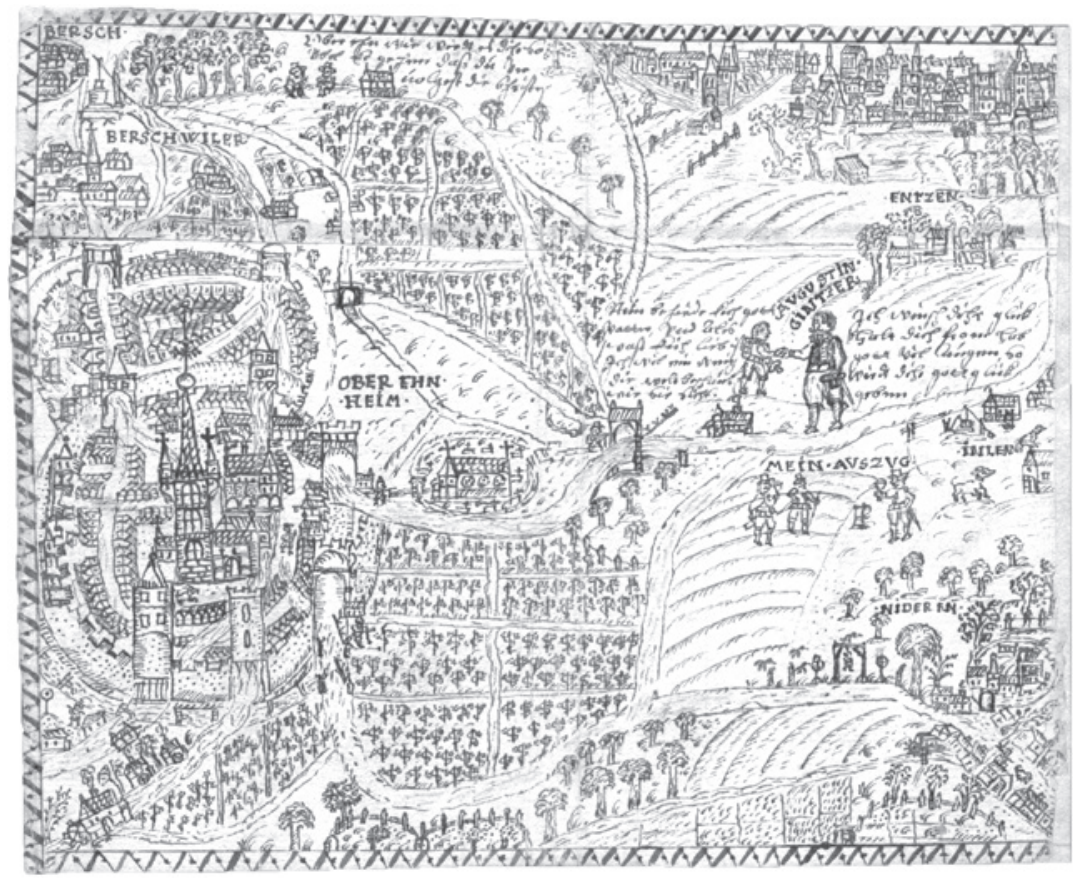

Fig. 2 - Départ d'Augustin Güntzer (Oberehnheim) comme compagnon potier d'étain, août 1615 (dessin d'Augustin Güntzer)

menace de soldats catholiques de la région lyonnaise ${ }^{10}$. Or, dans la suite de son récit, lorsque la marginalisation sociale - à son arrivée à Bâle commence à remplacer la marginalité religieuse, Güntzer se voit de plus en plus comme une espèce de martyr pour sa foi, se comparant fréquemment à Job. Cette tendance finit par s'inscrire dans son écrit, comme chez Erb, en l'occurrence dans le signe de la croix qu'il place au début de son "petit livre» («Büchlein»), et qui signifie notamment qu'il a vécu sous la croix.

Sans cesser de prendre en compte le fait que nous connaissons beaucoup plus de textes autobiographiques germanophones de provenance protestante que de textes catholiques, et donc que la prudence s'impose, j'ai l'impression qu'il existe une autre spécificité, à savoir un lien typiquement protestant entre la mémoire et la haine. Je ne songe pas ici aux préjugés confessionnels, que l'on rencontre un peu partout, mais plutôt

Io. A. Güntzer, Kleines Biechlin von meinem gantzen Leben, p. 196 et p. 144 (Sienne). 
à des expressions de haine visant des personnes spécifiques et résultant de mauvaises expériences des scripteurs. Il s'agit d'un phénomène que j'ai rencontré non pas uniquement, mais avant tout dans des textes protestants. On trouve une sorte d'archétype de ce mode particulier d'expression dans les notes autobiographiques de George Fox (1624-1691), fondateur du mouvement religieux des Quakers en Angleterre au milieu du XVII e siècle. L'auteur s'y montre très satisfait de ce que Dieu punisse régulièrement ceux qui le calomnient ${ }^{11}$.

Dans l'autobiographie rédigée dans les années 1650-1660 par l'architecte et conseiller de la ville d'Ulm Joseph Furttenbach (1596-1667), c'est son ancien associé, avec lequel il s'est brouillé, le marchand Hans Khon, qui est «victimisé» symboliquement sur le papier. Se félicitant de ce que le Bon Dieu lui ait offert la possibilité de faire de l'argent avec son cabinet de curiosités, Furttenbach relate avec quel chagrin il a remis par gain de paix 900 florins à son ancien partenaire commercial, avant de goûter à la satisfaction de la vengeance:

C'est ainsi qu'il [Hans Khon] a dû signer une déclaration reconnaissant qu'il n'avait plus rien à exiger de moi, quant à moi j'ai recommandé toute cette histoire à la vengeance divine [...]. Et voici que, sans que je n'aie rien fait pour cela, le bon juge commença à se mêler de l'affaire, Dieu le Tout puissant affligeant le dénommé Khon d'une goutte continuelle [...] de sorte qu'il ne put plus sortir pendant plusieurs mois, et que sa femme et sa belle-mère au cœur de serpent se querellaient et se lamentaient en disant qu'elles auraient préféré le voir mort plutôt que vivant dans cet état... ${ }^{12}$

Certes, il convient de préciser une nouvelle fois qu'on trouve des traces de cette vision très vétéro-testamentaire de Dieu également dans des témoignages catholiques du XVII ${ }^{\mathrm{e}}$ siècle, tels les pamphlets exhortant les fidèles à mieux se conduire collectivement pour éviter de s'attirer le châtiment divin. Ceci dit, sur le plan de l'analyse d'écrits personnels, je considère une telle posture comme un trait spécifiquement protestant: un phénomène qui marque d'ailleurs, de façon bien sûr involontaire, une distance considérable par rapport à la théologie de la justification par la foi des premiers réformateurs.

II. G. Fox, The Journal of George Fox, p. 96, 133-140 et 178-180.

I2. J. Furttenbach, Lebenslauff, p. 274, traduit de l'original allemand. 


\section{Le providentialisme}

Mes recherches antérieures sur les écrits personnels anglosaxons m'ont permis de constater que l'invocation de la providence divine, ou "providentialisme» - terme que j'emprunte à la recherche anglaise concernant le puritanisme -, y était très répandu: qu'il s'agisse d'expliquer l'heureuse issue d'un accident, l'obtention inattendue d'argent en période de pénurie ou même de spéculations sur ce qui aurait pu arriver en l'absence de protection divine. Le journal personnel du marchand Samuel Jeake (1652-1699), de Rye (Sussex), en fournit des exemples éloquents. Ainsi, Jeake observe en date du 16 août 1693 qu'il manqua tomber dans une rue sombre à cause d'un tas de bûches qui entravaient son chemin: «Mais il plut à Dieu d'envoyer la lueur d'un éclair [...] juste pour me montrer le danger imminent et pour l'écarter. Je ne cesserai de reconnaître cette providence. " ${ }^{13} \mathrm{Il}$ en va de même dans l'autobiographie de Barthold Hinrich Brockes, conseiller de la ville de Hambourg et auteur de nombreux poèmes qui célèbrent les traces que Dieu a laissées dans la nature. Brockes se souvient notamment qu'un hiver, à l'âge de douze ans environ, lui et sa sœur furent conduits en chariot bien fermé par les domestiques de la maison vers le bassin gelé de l'Alster au milieu de la ville. Quand les domestiques voulurent vérifier la solidité de la glace, ils s'enfoncèrent dans l'eau, avant de parvenir à s'en extraire: une grâce, note l'auteur, "qui ne se serait pas offerte à nous, les enfants, qui étions tout enveloppés, de sorte que nous aurions été incapables de nous aider et que nous nous serions noyés. Dieu soit loué!» ${ }^{14}$.

On trouve également des évocations de la providence divine dans les premières parties des notes autobiographiques du médecin Johann Heinrich Jung-Stilling (1740-1817) ${ }^{15}$ qui documentent la phase piétiste de sa vie, avant que le scripteur ne rejoigne le groupe des adhérents aux Lumières. Ceci dit, le providentialisme ne se révèle en aucun cas aussi prédominant, côté germanophone, que dans nombre de textes anglais du $\mathrm{XVII}$ siècle. Pourquoi? Bien que je me méfie des explications purement fonctionnalistes, je pense que cette différence a quelque chose à voir avec

I3. S. Jeake, An Astrological Diary of the Seventeenth Century, p. 234 sq. (August 16, 1693), traduit de l'anglais.

I4. B. H. Brockes, «Selbstbiographie», p. 4, traduit de l'allemand.

I5. J. H. Jung-Stilling, Lebensgeschichte. 
l'importance du dogme de la prédestination au sein de l'Eglise anglaise des $\mathrm{XVI}^{\mathrm{e}}$ et XVII ${ }^{\mathrm{e}}$ siècles, et surtout au sein du puritanisme. Le providentialisme aidait à relativiser le poids du dogme de la prédestination dans la vie individuelle parce qu'il tendait à rendre visible la présence de Dieu ici-bas, d'un Dieu dont les décisions quant au sort du croyant demeuraient insondables selon la théorie de la prédestination ${ }^{16}$. Que les Anglais du XVII e siècle aient très souvent choisi d'anthropologiser la providence divine en parlant de providences (au pluriel) adaptées aux cas individuels, et qu'ils aient établi une distinction entre good providences et bad providences va tout à fait dans le même sens.

Dans l'écriture de soi protestante du continent il n'est possible d'observer ce genre de tendances que dans des journaux intimes et autobiographies fortement influencés par le piétisme. Celui de Souabe tout comme le piétisme tardif de Bâle, de la première moitié du XIX ${ }^{\mathrm{e}}$ siècle, connaissait bien la pratique du Däumeln: en fermant les yeux, on mettait le doigt sur une page de la Bible, dans la croyance que la providence divine le dirigeait, de sorte que le passage de l'Ecriture Sainte ainsi trouvé était pris pour une sorte d'ordre du jour ${ }^{17}$.

Sur un plan plus général, les mentions de la providence divine demeuraient évidemment un appui important des textes protestants. L'autobiographie de Johannes Erb, évoquée ci-dessus, en est un bon exemple. A l'instar de nombreux textes de l'époque, elle est d'abord une démonstration de la présence active de Dieu dans les vies individuelles et collectives. C'est aussi le cas, par exemple, dans l'autobiographie de Joseph Furttenbach, jusque dans ses passages les moins séduisants, relatifs à la haine qu'il ressentait envers Johann Khon. Car selon Furttenbach, c'est bien Dieu lui-même qui punit Khon par ses attaques de goutte.

16. K. von Greyerz, «Biographical Evidence on Predestination, Covenant and Special Providence», p. 279. Voir aussi M. C. Jacob, M. Kadane, «Missing, Now Found in the Eighteenth Century», p. 20, note 3.

I7. S. Brückner, "Der "Frommen Lotterie" ". Voir aussi U. Bruckner-Eglinger, "Heute war ich bey Lisette in der Visite". 


\section{La lecture religieuse}

Autobiographies, journaux et chroniques familiales contiennent assez souvent des références à la pratique de l'écriture et de la lecture. Pour ce qui est de la lecture, les références à la Bible et à des traités d'édification spirituelle sont les plus fréquentes. Lorsqu'on aborde un tel aspect il convient toutefois, là aussi, de réaliser que nous avons affaire à des textes qu'il faut interpréter avec prudence. Sans partager l'opinion selon laquelle l'origine discursive des écrits personnels ne nous autoriserait à les examiner qu'à titre de fictions, il faut admettre que ce genre de documents pose des problèmes d'interprétation non négligeables.

Prenons, par exemple, le journal personnel de Barbara Schultheiss (1765-1792). Avant sa mort précoce, à l'âge de vingt-sept ans, la jeune femme appartenait au cercle d'admirateurs qui s'était formé à Zurich autour du pasteur et écrivain Johann Caspar Lavater, un personnage clé du piétisme tardif. Dans ce groupe, on ne négligeait certainement pas la lecture religieuse. Or Barbara Schultheiss n'en fait aucune mention, ne signalant que ses lectures profanes ${ }^{18}$. Il en va tout autrement du journal d'une autre Zurichoise, Regula von Orelli-Escher (1757-1829) ${ }^{19}$, plus jeune mais appartenant au même cercle, qui renvoie fréquemment à la lecture de textes religieux. Il semble difficile de croire que Barbara Schultheiss n'ait pas, elle aussi, lu de temps à autre dans la Bible. Ce genre de lecture relativement ritualisé allait-il tellement de soi qu'elle n'a pas jugé utile de le mentionner? Je n'ai pas de réponse convaincante à proposer à cette question, mais il est évident qu'il y a des pièges à éviter dans l'interprétation historique de l'écriture de soi.

Ces précautions prises, il convient de rappeler que la mention de la lecture religieuse est très fréquente dans les textes de protestants. Je me limiterai à un petit nombre d'exemples en ce qui concerne les XVI et XVII ${ }^{\mathrm{e}}$ siècles. Dans un passage important de son autobiographie, Georg Frell (1530-1597), un relieur anabaptiste, se souvient comment son père, mort en 1545, homme aux moyens particulièrement modestes, accumula des heures de travail supplémentaires afin de pouvoir acheter une Bible pour les siens, et surtout pour son fils, seul membre de la famille à savoir lire. Frell rapporte que la lecture dans la Bible n'a cessé depuis

I8. C'est ce que suggère le mémoire de licence inédit de B. Gügler, Lesen, p. 27.

I9. R. von Orelli-Escher, Selbstzeugnisse aus dem Umfeld von J.C. Lavater. 
lors d'être pour lui une source de joie et un agréable passe-temps ${ }^{20}$. Hans Ardüser (1557-1614), peintre et maître d'école dans les Grisons, nous fait savoir dans un passage de son autobiographie relatif à 1593 que ses dernières lectures se sont principalement concentrées sur des textes historiques (il est du reste l'auteur d'une chronique inédite). Pourtant la longue liste de titres qu'il insère dans un compte rendu dédié à cette même année mentionne en premier lieu la Bible. Elle est certes accompagnée de bon nombre de chroniques et textes historiques, mais également des "Cent sermons sur l'apocalypse de Jean", du "Grand livre des martyrs", de livres du réformateur Caspar Schwenckfeld, sans compter un "grand livre d'histoires diverses à propos des dix commandements " ${ }^{21}$. Quant au conseiller et architecte de la ville impériale d'Ulm déjà évoqué plus haut, Joseph Furttenbach, il nous introduit à la pratique très répandue chez les pieux protestants consistant à remplir un cahier après l'autre d'extraits tirés de la Bible: aussi bien une forme de méditation qu'un système mnémotechnique.

Outre ces cas d'auteurs laïcs, il conviendrait de mentionner l'écriture de soi de pasteurs protestants. Ces derniers se réfèrent régulièrement à la Sainte Ecriture. Pour ne donner que deux exemples, Johann Heinrich Hummel (1611-1674), originaire de Brugg en Argovie et plus tard doyen des pasteurs de la ville de Berne, commence son autobiographie en invoquant trois psaumes et, plus tard, mentionne des passages de la Bible auxquels il venait de consacrer des sermons ${ }^{22}$. Tandis qu'Alexander Bösch (1618-1693), pasteur réformé à Krummenau dans le Toggenbourg, loue la bonne éducation qu'il doit à ses parents en ces termes:

Ils m’ont envoyé à temps à l'école, pour que j’apprenne à lire et à écrire. J'ai appris beaucoup de beaux psaumes et versets avec pas grand chose, et je devais souvent les faire dire et prier aux gens qui venaient à la maison. J'ai beaucoup lu, dans toutes sortes de livrets, et tout particulièrement dans la Bible $[\ldots]^{23}$.

20. G. Frell, «Die Autobiographie des Täufers Georg Frell von Chur», p. 458 sq.

2I. P. Zinsli, Der Malerpoet Hans Ardüser, p. 141 et 145 sq. (liste de 1593).

22. J. H. Hummel, «Histori des Lebens Johannis Henrici Hummelii», p. 26, 38, 40 et 42 .

23. A. Bösch, Liber familiarium personalium, p. 73, traduit de l'original allemand. 
On peut se demander dans quelle mesure l'analyse d'écrits personnels confirme l'idée courante de l'importance particulière de la lecture religieuse chez les protestants. Ou, pour le formuler autrement, y a-t-il des différences confessionnelles à l'égard de la représentation autobiographique de la lecture religieuse? La question mériterait une étude systématique et approfondie. Je n'y apporterai que des éléments de réponse provisoires, sur la base de l'examen de dix écrits personnels catholiques rédigés entre le $\mathrm{XVI}^{\mathrm{e}}$ et le début du XIX ${ }^{\mathrm{e}}$ siècle. Ces textes sont aussi hétérogènes quant à leur genre que ceux de provenance protestante précédemment mentionnés. Pour chacun d'entre eux, il conviendrait de prendre plus particulièrement en compte les objectifs du texte, le public visé et les circonstances extérieures qui l'entourent, ce qui dépasse bien évidemment le cadre de mon propos.

Les journaux qui enregistrent essentiellement mariages, baptêmes, délits, phénomènes météorologiques exceptionnels et événements locaux, tel celui tenu entre 1559 et 1574 par l'officier épiscopal wurzbourgeois Adam Kahl ne contiennent pas d'indications sur des livres ou des lectures en raison de leur genre ${ }^{24}$. Les notes plus détaillées du conseiller et bourgmestre de Fulda Gangolf Hartung, de la première moitié du XVII siècle, sont quant à elles entièrement imprégnées par la guerre de Trente Ans et par ses répercussions à Fulda et dans les environs ${ }^{25}$. Il en va de même pour les deux témoignages conventuels consultés. Dans le volumineux journal de Klara Staiger, abbesse du couvent des Augustines de Mariastein, près d'Eichstätt, on trouve mention en date du dimanche de la Trinité (26 mai) de l'an 1641 des «livres des processions» dans lesquels est précisément fixé le déroulement de la litanie Surgite. A un autre endroit sont évoqués deux volumes contenant des vies de saints que deux sœurs ont acquis à Munich avec l'argent des aumônes ${ }^{26}$. On cherche en vain d'autres références en lien avec notre thème. Centrant le propos, à l'instar du journal de Klara Staiger, sur l'expérience de la violence effectuée pendant la guerre de Trente Ans, les terribles souffrances et les brutalités endurées, les notes du père bénédictin Johannes Bozenhart ${ }^{27}$

24. A. Kahl, Der Würzburger Adam Kahl (1539-1594) und sein Tagebuch (1559-1574).

25. G. Hartung, «Die chronikalischen Aufzeichnungen des Fuldaer Bürgers Gangolf Hartung (1607-1666)».

26. K. Staiger, Klara Staigers Tagebuch, p. 279 et 301.

27. J. Bozenhart, "Schicksale des Klosters Elchingen und seiner Umgebung in der Zeit des Dreißigjährigen Krieges (1629-1645)», p. 191 sq., 203 sq. et 215. 
ne contiennent, elles aussi, que deux références en lien avec ce qui nous intéresse ici. La première porte sur des livres qu'un prêtre d'Aislingen offre au couvent d'Elchingen, vers Neu-Ulm, en échange d'un peu de blé; la seconde concerne " 25 grands tomes» (" 25 grosse tomos») de la bibliothèque du couvent dont le seigneur de Leipheim prendrait volontiers possession en tant que contrepartie d'un prêt, ce qui lui est toutefois refusé. Dans une perspective analogue, la chronique de Stausebach, dans le nord de la Hesse, rédigée par le paysan catholique Caspar Preis pour les années 1636 à 1667 s'inscrit en réaction à la guerre de Trente Ans: la lecture religieuse n'y apparaît pas davantage que dans les textes mentionnés précédemment.

\section{Le cardinal von Harrach et ses lectures}

Qu'en est-il d'écrits personnels de provenance catholique qui ne se rattachent pas en premier lieu au contexte de la guerre de Trente Ans? L'examen du journal tenu entre 1566 et 1577 par le greffier de la ville de Bremgarten, dans l'Argovie suisse, Werner Schodeler le Jeune ${ }^{28}$, à l'instar de celui du livre de famille (Hausbuch) de la fin du XVII e siècle rédigé par Maria Elisabeth Stampfer de Vordernberg en Steiermark amène à des résultats tout aussi négatifs ${ }^{29}$. Et, aussi surprenant que cela puisse paraître, l'étude des diaires et des billets journaliers (Tagzettel) composés par le cardinal Ernst Adalbert von Harrach (1598-1667) ne mène pas à des conclusions fondamentalement différentes.

Les sept épais volumes parus en 2010 de ce qui constitue à ma connaissance le plus long écrit personnel du XVII siècle $^{30}$ révèlent un scripteur à l'évidence prolixe, qui a en outre, comme le montrent plusieurs passages de son texte, reçu un nombre particulièrement élevé de lettres. Encore en cours - mais néanmoins représentative au terme de plusieurs semaines de confrontation avec ce texte - mon analyse aboutit à la conclusion que si

28. W. Schodeler, «Wernher Schodelers des jüngern Tagebuch».

29. M. E. Stampfer, Maria Elisabeth Stampfer aus Vordernberg.

30. E. A. von Harrach, Die Diarien und Tagzettel des Kardinals Ernst Adalbert von Harrach (1598-1667). Voir mon compte rendu dans Mitteilungen des Instituts für Österreichische Geschichtsforschung, 120 (2012), p. 173 sq. Je me limite, dans les considérations qui suivent, à l'examen des billets journaliers, les diaires, rédigés en italien, présentant un aspect plus formalisé de journal de fonction. 
l'auteur évoque à plusieurs endroits des pratiques religieuses et ecclésiastiques d'époque et qu'il lisait d'innombrables messes, on ne rencontre guère sous sa plume de références concrètes à la lecture religieuse.

De quoi est-il donc question lorsqu'il en va de livres dans les billets journaliers du cardinal? Le 9 mars 1638, le prélat indique qu'il a envoyé un messager de sa terre de Retschitz (Cervena Recice) au colonel Maximilian von Steegen, pour aller quérir les livres français qui lui ont été promis, visiblement destinés aux femmes et autres hôtes hébergés dans son château, conformément à la culture de la sociabilité entretenue par la noblesse bohême. Au nombre de ces lectures occasionnelles figurent également des calendriers au contenu astrologique, que le scripteur évoque, en passant, plus d'une décennie et demie plus tard: "Les nouveaux calendriers nous prédisent pour cette année plutôt du bon que du mauvais en matière de guerre. " ${ }^{31} \mathrm{Ou}$ encore des "histoires" ("Historien») dont il ne précise pas le contenu.

On peut dès lors considérer comme une énorme chance de pouvoir lire un passage tel que celui qui se rapporte au dernier retour du cardinal du quatrième conclave romain auquel il ait participé, en date du 24 septembre 1667 , un mois avant sa mort. Des raisons de santé contraignent alors le prélat à voyager dans une litière. Il en relève le principal inconvénient, "le défaut de conversation", auquel, après une heure ou une heure et demie de prière, il pallie par la lecture:

Je lis alors quelque chose d'amusant, et quand cela me fatigue je change d'ouvrage, j'en prends un autre qui soit plus drôle, ou même un roman [Romance], et si le sommeil me gagne je lui donne aussi audience, tant qu'il le souhaite ${ }^{32}$.

Ernst Adalbert von Harrach ne se bornait pas à lire dans sa litière. Il le faisait aussi lorsqu'il voyageait en carrosse, toujours «à défaut de conversation». Fin octobre 1655, en route pour la Bohême, il rapporte - à la troisième personne - depuis un village proche de Rokizan (Rokycany), que «le brouillard survenu en cours de route a tant contraint le cardinal à la lecture qu'il manqua peu pour qu'il en devint aveugle» ${ }^{33}$. Mais on n'apprendra malheureusement rien de plus concret sur la lecture en question.

3I. Ibid., vol. 6, p. 96 (17. Oktober 1655, Prag), traduit de l'original allemand.

32. Ibid., vol. 7, p. 894 (Serravalle di Chienti), traduit de l'original allemand.

33. Ibid., vol. 6, p. 100, traduit de l'original allemand. 


\section{Représentations d'une pratique}

Pour conclure ce survol de textes émanant de scripteurs catholiques issus de milieux très divers, je me suis penché sur la chronique conjugale et familiale du maître charpentier Balthasar Joseph Tschudi d'Ennenda, dans le canton suisse de Glaris, mort en 1807. La décapitation de Louis XVI et surtout les guerres napoléoniennes sur le sol helvétique et dans sa propre localité y prennent une place importante, à côté des affaires domestiques et d'observations détaillées sur les onze grossesses et accouchements douloureux de son épouse Maria Magdalena ${ }^{34}$. Des références directes à la Bible ou à d'autres lectures religieuses n'apparaissent qu'à deux reprises dans ce texte qui se veut très exhaustif. La première consiste en une référence négative à Esaü, qui n’aurait pas demandé le consentement de ses parents pour se marier. La seconde renvoie au récit du rêve de «Joseph l'Egyptien» qui aurait eu des connaissances en la matière. Marquée par nombre de répétions, cette éloquente chronique du couple et de la maisonnée destinée aux enfants laisse à divers endroits deviner entre les lignes des connaissances bibliques, quand bien même la lecture de la Bible ou celle d'histoires bibliques ne sont jamais thématisées.

Le protestantisme de l'époque moderne a été non sans raison souvent qualifié de «religion du livre». On ne saurait, sur la base des écrits personnels examinés ici, dire la même chose du catholicisme germanophone des temps modernes. C'est un constat que j'avance avec toute la prudence requise par le faible échantillon et par la grande hétérogénéité de genre des textes analysés ici. Il serait bien sûr erroné de déduire de façon positiviste des écrits de scripteurs et scriptrices catholiques en question que la lecture religieuse ne jouait guère de rôle dans leur vie. Il est important de se souvenir du fait que nous ne parlons pas d'une pratique en tant que telle, mais plutôt de la représentation d'une certaine pratique. Ce sont surtout ces considérations contextuelles qui nous imposent la conclusion que les auteurs protestants jugeaient leur lecture religieuse d'une importance qui assurait à cette pratique des mentions fréquentes dans les textes en question tandis que tel n'était pas le cas dans la composition de textes autobiographiques de provenance catholique. Ce que les dix textes catholiques envisagés laissent supposer, c'est donc que la lecture

34. B. J. Tschudi, «Chronik der Familie des Balthasar Joseph Tschudi von Ennenda». 
religieuse n'y était pas considérée comme aussi digne d'être communiquée que dans les textes d'auteur-e-s protestant-e-s.

Mieux étudiée dans le cas de la France que pour l'espace germanophone, la lecture religieuse a bien sûr aussi existé dans les territoires catholiques de langue allemande. Il suffit à cet égard de songer à l'histoire de la Contre-Réforme, telle qu'elle apparaît ponctuellement dans les notes malheureusement seulement en partie conservées du Bavarois Abraham Kern. L'auteur y décrit une perquisition d'ouvrages systématique, inspirée par la Contre-Réforme et dirigée par le conseil aulique munichois, qui se déroula en novembre 1608 dans sa ville natale de Wasserburg: "Les ouvrages suspects [furent] saisis, avec les noms des hommes et des femmes auxquels ils appartenaient inscrits dedans, portés au château princier et ensuite envoyés à Munich.» ${ }^{35}$ On ignore malheureusement les suites de cette perquisition. Mais on sait, par d'autres exemples, que de telles confiscations pouvaient aller très (trop) loin. L'humaniste Rudolf Collinus (1499-1578) un des adhérents précoces de la Réforme protestante en Suisse centrale se souvient dans son autobiographie que le conseil de Lucerne alla jusqu'à confisquer l'un de ses livres en grec, un membre du conseil, qui ne maîtrisait pas cette langue, ayant déclaré: "Was Kritzis Kretzis ist, das ist lutherisch!», soit «ce qui est illisible et inexplicable est bien luthérien ${ }^{36}$.

\section{Protestantisme européen et écriture de soi: remarques conclusives}

En guise de conclusion, on relèvera d'abord que contrairement aux protestantismes germanophones, le puritanisme anglais a généré depuis la fin du $\mathrm{XVI}^{\mathrm{e}}$ siècle une riche variété d'autobiographies et de journaux personnels à caractère spirituel. La méditation religieuse, la construction identitaire du groupe des pieux et l'autodiscipline y jouent un rôle important ${ }^{37}$. Cette dernière s'applique en premier lieu au contrôle de l'usage du temps passé dans l'ici-bas. L'appel «to redeem time» qu'on rencontre partout dans ce

35. A. Kern, "Aus dem Tagbuch des Abraham Kern von Wasserburg», p. 160, traduit de l'allemand. Le journal porte principalement sur la période 1579-1628. Seuls des extraits en ont été publiés, l'original semblant, quant à lui, avoir disparu.

36. R. Collin, «Rudolf Collins Schilderung seines Lebens».

37. K. von Greyerz, "L'autoformation spirituelle en Angleterre (XVII ${ }^{\mathrm{e}}$ siècle)». 
genre de documents est très révélateur. Il est évident qu'il y a des liens directs entre la notion de "rédemption (du temps) » et le dogme réformé de la prédestination. Mais, dans ce cas, on peut se demander pourquoi cette forme spécifique d'écriture spirituelle ne s'est pas répandue aussi parmi les huguenots français. C'est ce qu'a fait Philip Benedict ${ }^{38}$. Lauteur explique la différence entre la tradition anglaise et celle des huguenots français de la façon suivante. Côté anglais, les puritains n'étant jamais parvenus à se mettre d'accord sur une discipline ecclésiastique unifiée, il fallait bien que les fidèles s'imposent une forme d'autodiscipline qu'ils trouvaient dans le journal et l'autobiographie spirituels, tandis qu'en France les huguenots réussirent à introduire des consistoires paroissiaux sur le modèle genevois, ce qui les aurait libérés, toujours selon cette interprétation, de la nécessité d'un auto-contrôle.

L'explication est sans conteste séduisante. Elle n'en demeure pas moins hautement fonctionnaliste, dans le sens où elle établit une relation très étroite à l'intérieur d'une sorte de triangle entre ce genre d'écriture de soi, la discipline morale et le dogme de la prédestination. C'est ce que j'appellerais une lecture "weberienne", et Philip Benedict se réfère du reste très directement à Max Weber et à sa thèse sur l'ascétisme particulier des calvinistes, quand bien même il finit par parler de deux calvinismes distincts. A la lumière des recherches récentes dans ce champ qui nous est commun, je pense toutefois qu'il conviendrait d'aller plus loin, et de relativiser jusqu'à un certain point l'accent mis dans l'interprétation sur l'individu et l'individualisation spirituelle. Chaque tradition régionale ou nationale de l'écriture de soi s'insère dans un contexte culturel concret qui est un véhicule de notions identitaires au-delà du dogme religieux. En refusant de l'admettre on éprouve quelque peine, par exemple, à comprendre pourquoi Samuel Pepys, l'auteur bien connu d'un long journal personnel dans les années 1660, se sanctionne souvent pour un mauvais usage de son temps bien qu'il ne soit certainement pas puritain. C'est dire à quel point il est difficile de fonctionnaliser la diversité des langages autobiographiques.

Kaspar von Greyerz

Université de Bâle

Traduit de l'allemand par Danièle Tosato-Rigo

38. P. Benedict, "Two Calvinisms». 


\section{BIBLIOGRAPHIE}

\section{Sources}

Bösch, Alexander, Liber familiarium personalium, das ist, Verzeichnis waß sich mit mir, und der meinigen in meiner hausshaltung, sonderliches begeben und zugetragen hatt. Lebensbericht und Familiengeschichte des Toggenburger Pfarrers Alexander Bösch (1618-1693), hrsg. von Lorenz Heiligensetzer, Basel, Schwabe, 2001 (Selbst-Konstruktion, 1).

Bozenhart, Johannes, "Schicksale des Klosters Elchingen und seiner Umgebung in der Zeit des Dreißigjährigen Krieges (16291645). Aus dem Tagebuche des P. Johannes Bozenhart", hrsg. von P. Luitpold Brunner, Zeitschrift des Historischen Vereins für Schwaben und Neuburg, 3 (1876), p. 157-282.

Brockes, Barthold Heinrich, "Selbstbiographie", in Selbstbiographie. Verdeutschter Bethlehemitischer Kinder-Mord, Gelegenheitsgedichte, Aufsätze, hrsg. von Jürgen Rathje, Göttingen, Wallstein, 2012, p. $1-40$.

Bruckner-Eglinger, Ursula, "Heute war ich bey Lisette in der Visite». Die Tagebücher des Basler Pfarrersfrau Ursula Bruckner-Eglinger, 1816-1833, hrsg. von Bernadette Hagenbuch, Basel, Schwabe, 2014 (Selbst-Konstruktion, 6).

Collin, Rudolf, "Rudolf Collins Schilderung seines Lebens", hrsg. von Salomon Vögelin, Zürcher Taschenbuch auf das Jahr, 1859, p. 179-220.

Fox, Georg, The Journal of George Fox, ed. by John L. Nickalls, Cambridge, Cambridge University Press, 1952.

Frell, Georg, "Die Autobiographie des Täufers Georg Frell von Chur", hrsg. von Simon Rageth, Zwingliana, 7 (1939-1943), p. 444-469.

Furttenbach, Joseph, Lebenslauff, hrsg. von Kaspar von Greyerz, Kim Siebenhüner, Roberto Zaugg, Köln/Weimar/Wien, Böhlau, 2013 (Selbstzeugnisse der Neuzeit, 22). 
GüntZer, Augustin, Kleines Biechlin von meinem gantzen Leben. Die Autobiographie eines Elsässer Kannengießers aus dem 17. Jahrhundert, hrsg. von Fabian Brändle, Dominik Sieber, Köln/ Weimar/Wien, Böhlau, 2002 (Selbstzeugnisse der Neuzeit, 8).

-, L'histoire de toute ma vie. Autobiographie d'un potier d'étain calviniste du XVII e siècle, éd. par Fabian Brändle, Dominik Sieber, trad. de l'allemand par Monique Debus Kehr, Paris/Genève, Champion / Slatkine, 2010 (Vie des Huguenots, 55).

Harrach, Ernst Adalbert von, Die Diarien und Tagzettel des Kardinals Ernst Adalbert von Harrach (1598-1667), hrsg. von Katrin Keller, Alessandro Catalano, 7 Bde., Köln/Wien/Weimar, Böhlau, 2010 (Veröffentlichungen der Kommission für Neuere Geschichte Österreichs, 104).

Hartung, Gangolf, "Die chronikalischen Aufzeichnungen des Fuldaer Bürgers Gangolf Hartung (1607-1666) », hrsg. von Theodor Haas, Fuldaer Geschichtsblätter, 9 (1910), p. 49-126, 129-171.

Hummel, Johann Heinrich, "Histori des Lebens Johannis Henrici Hummelii", hrsg. von Christian Erni, Berner Zeitschrift für Geschichte und Heimatkunde, 1950, p. 24-57.

Jeake, Samuel, An Astrological Diary of the Seventeenth Century. Samuel Jeake of Rye, 1652-1699, ed. by Michael Hunter, Annabel Gregory, Oxford, Oxford University Press, 1988.

Jung-Stilling, Johann Heinrich, Lebensgeschichte, hrsg. von Gustav Adolf Benrath, Darmstadt, Wissenschaftliche Buchgesellschaft, 1992.

Kahl, Adam, Der Würzburger Adam Kahl (1539-1594) und sein Tagebuch (1559-1574), hrsg. von Heinrich Endres, Wilhelm Engel, Würzburg, Freunde mainfränkischer Kunst und Geschichte, 1952 (Mainfränkische Hefte, 14).

Kern, Abraham, "Aus dem Tagbuch des Abraham Kern von Wasserburg", in Beyträge zur vaterländischen Historie, Geographie, Statistik und Landwirthschaft, samt einer Uebersicht der schönen Literatur, hrsg. von Lorenz Westenrieder, München, Lindauer, 1788, Bd. 1, p. 146-173.

Kölderer, Georg, Beschreibung vnnd Kurtze Vertzaichnus Fürnemer Lob vnnd gedenckhwürdiger Historien. Eine Chronik der Stadt Augsburg der Jahre 1576 bis 1607, bearbeitet von Silvia Strodel, hrsg. von 
Wolfgang E. J. Weber, Augsburg, Wissner, 2013 (Documenta Augustana, 26).

Orelli-Escher, Regula von, Selbstzeugnisse aus dem Umfeld von J. C. Lavater, hrsg. von Gustav W. von Schulthess, Stäfa, Th. Gut, 2001.

Schodeler, Wernher, "Wernher Schodelers des jüngern Tagebuch", hrsg. von Walther Merz, Taschenbuch der historischen Gesellschaft des Kantons Aargau für das Jahr, 1904, p. 77-164.

Staiger, Klara, Klara Staigers Tagebuch. Aufzeichnungen während des Dreißigjährigen Krieges im Kloster Mariastein bei Eichstätt, hrsg. von Ortrun Fina, Regensburg, Friedrich Pustet, 1981.

Stampfer, Maria Elisabeth, Maria Elisabeth Stampfer aus Vordernberg: Hausbuch, hrsg. von Joseph von Zahn, Wien, A. Hölder, 1887.

Tschudi, Balthasar Joseph, "Chronik der Familie des Balthasar Joseph Tschudi von Ennenda und seiner Frau Maria Magdalena Stählin von Netstal (begonnen am 20. Christmonat 1790, beendet nach 1802)", hrsg. von German Studer-Freuler, Jahrbuch des Historischen Vereins des Kantons Glarus, 76 (1996), p. 11-146.

Zillhardt, Gerd, Der Dreissigjährige Krieg in zeitgenössischer Darstellung. Hans Heberles "Zeytregister" (1618-1672). Aufzeichnungen aus dem Ulmer Territorium: ein Beitrag zur Geschichtsschreibung und Geschichtsverständnis der Unterschichten, Stuttgart, Kohlhammer Komm, 1975 (Forschungen zur Geschichte der Stadt Ulm, 13).

\section{Travaux}

Benedict, Philipp, "Two Calvinisms", in The Faith and Fortunes of France's Huguenots, 1600-85, Aldershot, Ashgate, 2001, p. 208228 (St Andrews Studies in Reformation History).

BRÜCKner, Shirley, "Der "Frommen Lotterie". Pietistische Lospraktiken in der Schweiz", Jahrbuch der Schweizerischen Gesellschaft für die Erforschung des 18. Jahrhunderts, 2 (2011), p. 66-87.

Greyerz, Kaspar von, "Biographical Evidence on Predestination, Covenant and Special Providence", in Weber's "Protestant Ethic”. Origins, Evidence, Contexts, ed. by Hartmut Lehmann, 
Guenther Roth, Cambridge/New York, Cambridge University Press, 1993, p. 273-284.

—, "L'autoformation spirituelle en Angleterre (XVII ${ }^{\mathrm{e}}$ siècle)", in Autodidaxies, $X V I^{e}-X I X^{e}$ siècles, éd. par Willem Frijhoff, Paris, Institut national de recherche pédagogique, 1996, p. 49-63.

GügLER, Brigitte, Lesen: Zu den Funktionen einer kulturellen Praxis am Ende des 18. Jahrhunderts. Eine Untersuchung des Tagebuchs von Anna Barbara Schulthess, verh. Gessner (1765-1792), mémoire de licence inédit, Univeristé de Zurich, 2002.

Heiligensetzer, Lorenz, Getreue Kirchendiener - gefährdete Pfarrherren. Deutschschweizer Prädikanten des 17. Jahrhunderts in ibren Lebensbeschreibungen, Köln/Weimar/Wien, Böhlau, 2006 (Selbstzeugnisse der Neuzeit, 15).

—, "Swiss-German Self-Narratives. The Archival Project as a Rich Vein of Research", in Mapping the "I". Research on Self Narratives in Germany and Switzerland, ed. by Claudia Ulbrich, Kaspar von Greyerz, Lorenz Heiligensetzer, Leiden, Brill, 2015, p. 58-75.

Holzem, Andreas, "Zeit - Zeitenwende - Endzeit? Anfangsbeobachtungen zum deutschen katholischen Schrifttum um 1700", in Jahrhundertwenden. Endzeit- und Zukunftsvorstellungen vom 15. bis zum 20. Jahrhundert, hrsg. von Manfred Jakubowski-Tiessen et al., Göttingen, Vandenhoeck \& Ruprecht, 1999, p. 213-232 (Veröffentlichungen des Max Planck Instituts für Geschichte, 155).

Jacob, Margaret C., Kadane, Matthew, "Missing, Now Found in the Eighteenth Century: Weber's Protestant Capitalist", The American Historical Review, 108 (February 2003), p. 20-49.

Schulz, Christian, "Das Strafgericht Gottes oder menschliches Versagen? Die Tagebücher des Benediktiberabtes Georg Gaisser als Quelle für die Kriegserfahrung von Ordensleuten im Dreissigjährigen Krieg", in Das Strafgericht Gottes. Kriegserfahrungen und Religion im Heiligen Römischen Reich Deutscher Nation im Zeitalter des Dreissigjährigen Krieges, hrsg. von Matthias Asche, Anton Schindling, Münster, Aschendorff, 2001, p. 219-290.

Sieber, Dominik, «Calvinistische Passionen, konfessionalisierte Körper. Zur Autobiographie des Zinngießers Augustin Güntzer (15961657 ?) ", Sozialwissenschaftliche Informationen, 24 (1995), p. 5-11. 
Tosato-Rigo, Danièle, La chronique de Jodocus Jost. Miroir du monde d'un paysan bernois au XVII siècle, Lausanne, SHSR, 2009.

Zinsli, Paul, Der Malerpoet Hans Ardüser. Eine volkstümliche Doppelbegabung um die Wende des 16. Jahrhunderts, Chur, Terra Grischuna, 1986.

\section{Crédits iconographiques}

Fig. 1 - La comète de 1618 menaçant le sud de l'Allemagne. Dessin à la plume dans: Augustin Güntzer, Kleines Biechlin... [Autobiographie], manuscrit, Bibliothèque universitaire de Bâle, HV, 165.

Voir aussi : http://dx.doi.org/10.7891/e-manuscripta-13484.

Fig. 2 - Départ d'Augustin Güntzer (Oberehnheim) comme compagnon potier d'étain, août 1615. Dessin à la plume dans: Augustin Güntzer, Kleines Biechlin... [Autobiographie], manuscrit, Bibliothèque universitaire de Bâle, HV, 165.

Voir aussi : http://dx.doi.org/10.7891/e-manuscripta-13484. 
